Peer Reviewed Paper openaccess Special Issue on Chemometrics in Hyperspectral Imaging

\title{
Estimation of phosphorus-based flame retardant in wood by hyperspectral imaging-a new method
}

\author{
Petter Stefansson, ${ }^{\mathrm{a}, *}$ Ingunn Burud, ${ }^{\mathrm{b}}$ Thomas Thiis, ${ }^{\mathrm{c}}$ Lone R. Gobakken ${ }^{\mathrm{d}}$ and Erik Larnøy \\ aFaculty of Science and Technology, Norwegian University of Life Sciences, Drøbakveien 31, 1430 Ås, Norway. \\ E-mail: petter.stefansson@nmbu.no \\ ${ }^{b}$ Faculty of Science and Technology, Norwegian University of Life Sciences, Drøbakveien 31, 1430 Ås, Norway. \\ ORCID: https://orcid.org/0000-0003-0637-4073 \\ 'Faculty of Science and Technology, Norwegian University of Life Sciences, Drøbakveien 31, 1430 Ås, Norway \\ ${ }^{d}$ Norwegian Institute of Bioeconomy Research, PO Box 115, 1431 Ås, Norway \\ eNorwegian Institute of Bioeconomy Research, PO Box 115, 1431 Ås, Norway. ORCID: https://orcid.org/0000-0002-8724-4010
}

It is recognised that flame retardant chemicals degrade and leach out of flame-protected wood claddings when exposed to natural weathering. However, the ability to survey the current state of a flame retardant treatment applied to a wood cladding, an arbitrary length of time after the initial application, is limited today. In this study, hyperspectral imaging in the near infrared to short-wavelength infrared region is used to quantify the amount of flame retardant present on wooden surfaces. Several sets of samples were treated with various concentrations of a flame retardant chemical and scanned with a push broom hyperspectral camera. An inductively coupled plasma (ICP) spectroscopy analysis of the outermost layer of the treated samples was then carried out in order to determine each sample's phosphorus content, the active ingredient in the flame retardant. Spectra from the hyperspectral images were pre-processed with extended multiplicative scatter correction, and the phosphorus content was modelled using a partial least squares (PLS) regression model. The PLS regression yielded robust predictions of surface phosphorus content with a coefficient of determination, $R^{2}$, between 0.8 and 0.9 on validation data regardless of whether the flame retardant chemical had been applied to the surface of the wood or pressure-impregnated into it. The result from the study indicates that spectral imaging around the $2400-2531 \mathrm{~nm}$ wavelength region is favourable for quantifying the amount of phosphorus-based flame retardant contained in the outermost layer of non-coated wooden claddings. The results also reveal that the uptake of phosphorus-based flame retardant does not occur uniformly throughout the wood surface, but is to a larger extent concentrated in the earlywood regions than in the latewood.

Keywords: hyperspectral imaging, NIR, flame retardant treated wood

\section{Introduction}

Throughout the European building sector wood is strengthening its position as a preferred building material in many applications. ${ }^{1}$ This trend is incentivised by the European Union's Europe 2020 targets, which aim to substantially decrease the greenhouse gas emissions and increase energy-efficiency in the EU by the year 2020, partly by promoting the sustainable use of wood in construction. ${ }^{2}$ Wood materials used in buildings already benefit from

\section{Correspondence}

Petter Stefansson (petter.stefansson@nmbu.no)

Received: 15 September 2017

Revised: 30 November 2017

Accepted: 31 January 2018

Publication: 15 February 2018

doi: $10.1255 /$ jsi.2018.a3

ISSN: 2040-4565

\section{Citation}

P. Stefansson et al., "Estimation of phosphorus-based flame retardant in wood by hyperspectral imaging-a new method", J. Spectral Imaging $\mathbf{7}$, a3 (2018). https://doi.org/10.1255/jsi.2018.a3

(c) 2018 The Authors

This licence permits you to use, share, copy and redistribute the paper in any medium or any format provided that a full citation to the original paper in this journal is given, the use is not for commercial purposes and the paper is not changed in any way. 
having a low carbon footprint, ${ }^{3}$ and through increased focus on material selection which is fit-for-purpose and by facilitating predictable long-term performance, their footprint can be reduced even further. However, the combustibility of wood can be a challenge when wood is used as a construction material. Due to the risk of fire, many European countries adopted an outright ban on constructing timber buildings taller than two stories for a long time. More developed building regulations and an increased understanding of fire safety has, however, in the last few decades caused many of these bans to be lifted. It is now both permitted and often encouraged to build multi-story timber buildings in most European countries. ${ }^{4}$ One effective strategy for increasing the fire safety of wood-based buildings is to use wood materials which prior to assembly in the construction phase, have been treated with a phosphorus-based flame retardant chemical. In an event of fire, such treatments react by producing a char surface which prevents the degradation of the wood and delays the point of flashover.

An important fire safety aspect, which currently is largely unknown, is how wood materials treated with flame retardant chemicals are affected by natural weathering and how the treatment withstands long-term exposure to the external climate. It is known that phosphorus, a crucial component in many flame retardant treatments, tends to leach out over time from the wood it has been impregnated into. ${ }^{5}$ By weighing a piece of lumber before and after it undergoes a chemical treatment to increase its flame resistance, it is possible to determine the total uptake of flame retardant compound in the wood. To determine the amount of chemical which resides in the outermost layer of a wood cladding treated with a flame retardant, an arbitrary length of time after the initial treatment, is considerably more difficult. Especially if it is to be done in a nondestructive way. For this reason, the ability to survey and inspect the treatment status of existing wooden constructions or stored lumber is severely limited.

In this paper, a new method for quantifying the current concentration of flame retardant in the outer layer of wood samples using near infrared (NIR) hyperspectral imaging is developed and evaluated. NIR spectroscopy is widely used for quality control in numerous fields, e.g., the food industry, ${ }^{6}$ and the potential for using NIR spectroscopy in the field of wood science has successfully been demonstrated in previous studies. ${ }^{7-9}$ NIR hyperspectral imaging has also been applied to wood surfaces to determine wood moisture content, ${ }^{10}$ map chemical composition, ${ }^{11}$ determine wood extractive content, ${ }^{12}$ map weathering by UV radiation, ${ }^{13}$ for identification of compression wood ${ }^{14}$ and for detecting show-through resin defects on painted lumber. ${ }^{15}$

The method proposed in this paper involves mapping the NIR absorbance spectrum of a wood sample, by means of a linear regression model, to its corresponding surface concentration of phosphorus. Phosphorus is the active ingredient in many flame retardant chemicals and can be seen as proportional to its ability to protect the wood against fire. By measuring the NIR spectra using hyperspectral imaging, it also becomes possible to spatially resolve the absorbance properties of the wood, which enables completely non-destructive and rapid surveying of large areas of wood such as facades or wooden decks.

\section{Method \\ Sample preparation and signal acquisition}

Five boards of Norway spruce (Picea abies), originating from different logs, were each cut into seven samples of dimension $50 \times 50 \times 10 \mathrm{~mm}$, giving a total of 35 samples. The samples were conditioned in a climate chamber at $20^{\circ} \mathrm{C}$ and $65 \%$ relative humidity until the samples had reached equilibrium moisture content. The samples were treated with Preventor AntiFlame from Akzo Nobel, which is a flame retardant wood coating commonly used in the construction industry. According to the Akzo Nobel, the undiluted chemical is typically diluted with distilled water to reach any desired solution concentration prior to applying it to wood, and a concentration of $66 \%$ is common when pressure-impregnating the chemical into wood. In our study, the Preventor AntiFlame was diluted with distilled water into seven different concentrations: 0 , 17, 33, 50, 67, 83 and 100\%. Each of the seven concentrations was then used to treat 5 of the 35 samples in a manner such that each concentration was represented once in each of the original wood logs. This was done to ensure that any model developed from the data set had the ability to reliably estimate the concentration of flame retardant chemical in the wood despite natural variations in the physical appearance of samples originating from different trees, which could affect their spectrum.

To ensure even and comparable uptake of each solution, the solutions were poured into petri dishes and each sample was immersed in the solution for 30 seconds. In 
addition to the immersed samples, five samples that were industrially pressure-impregnated using the same flame retardant chemical at a concentration of $66 \%$ were also included in the study to verify that the spectral fingerprint of the flame retardant substance is the same independent of treatment method.

A custom plastic sample mount was designed and 3D-printed, which could accommodate five samples at a time in addition to a Spectralon white reference plate during image acquisition. The samples were then placed inside the plastic mount, illuminated by two halogen lights and scanned using a push broom hyperspectral camera (Specim, Oulu, Finland) with 256 bands in the $929-2531 \mathrm{~nm}$ range, as can be seen illustrated in Figure 1.

After signal acquisition, a three-dimensional representation of the reflective properties of each sample was acquired, with spatial width along one dimension, spatial height along one dimension and reflected light along the third as can be seen illustrated in Figure 2. The spatial resolution of each image was $151 \times 151$ pixels, resulting in a pixel dimension of $0.33 \mathrm{~mm}^{2}$.

When the reflective properties of each sample had been measured, a 0.4-0.5 mm layer was removed from the surface of each sample using a planer, which was cleaned with alcohol between every sample to prevent cross contamination. The phosphorus content in the removed layers was analysed according to the ICP21100 Thermo Jarell Ash ICP-IRIS HR Duo method. ${ }^{16}$ The laboratory established surface concentration of phosphorus for each sample were used as response values when developing the regression model.

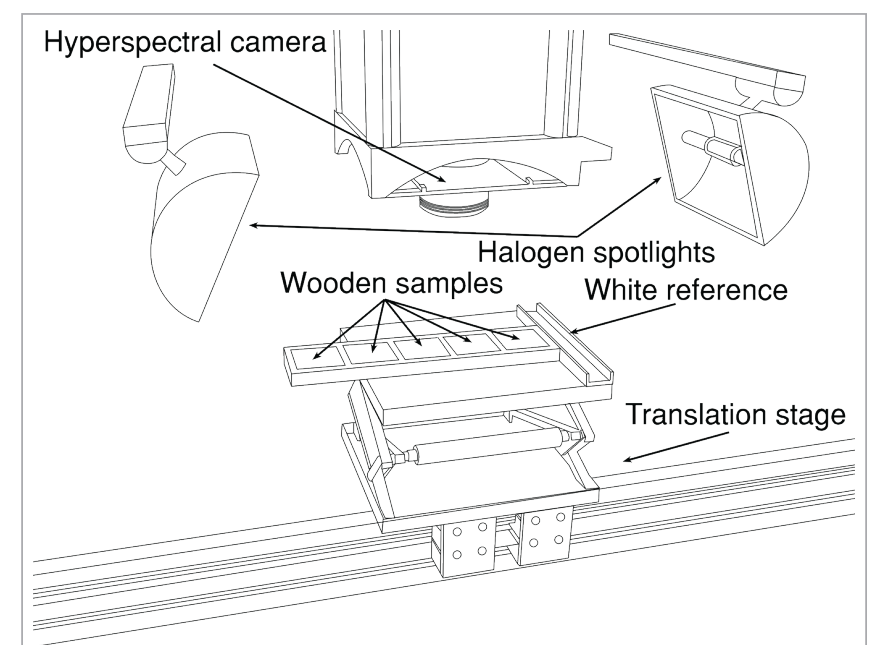

Figure 1. Illustration of experimental setup.

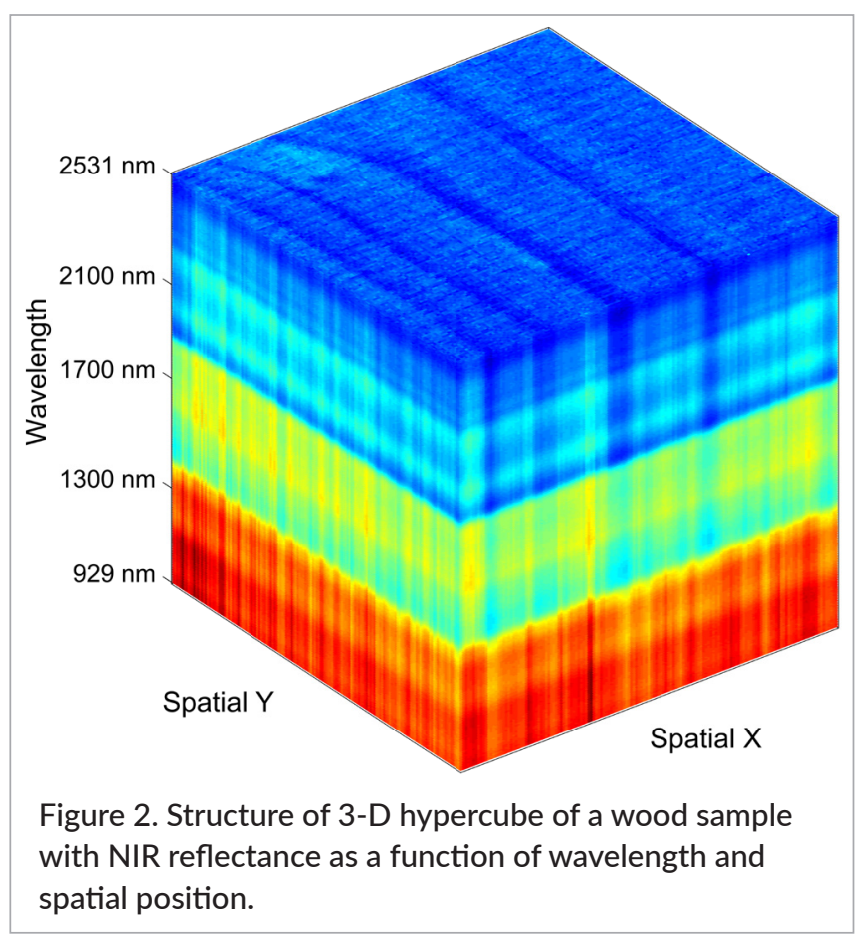

The hyperspectral data were divided into a training set (5/8 of the data) and a validation set (3/8 of the data). The samples treated with concentrations $0,33,50,83$ and $100 \%$ were chosen as training data to be used when developing the regression model. Samples treated with concentrations of $17 \%$ and $67 \%$, together with the pressure-impregnated samples, were consequently not used in the calibration of the regression model. These samples could therefore be used to validate the final model's ability to generalise to new unseen data and estimate the phosphorus content of new wood samples. One of the five samples treated with $17 \%$ chemical concentration was, however, later excluded from the validation data set since it was found to be covered in resin, which severely distorted its spectral properties. Table 1 shows a summary of all the samples in the data set and their treatment.

\section{Data pre-processing}

The signal from each hyperspectral image was first recalculated into reflectance, relative to a white reference, by subtracting the mean value of each pixel row in the spatial direction of a dark region (taken with the camera shutter closed), and dividing by the mean intensity value of a white calibration plate included in the image, also for each pixel row. The reflectance spectra, $R$, were transformed into absorbance, $A$, using the rela- 
Table 1. Overview of samples included in the study. Marooncoloured cells represent samples used as calibration data, whilst blue and orange cells indicate immersed, $\mathrm{T}$, and pressure-impregnated, IM, samples used to validate the PLS model's performance. The crossed-out cell represents a resin covered sample which was excluded from the validation data set.

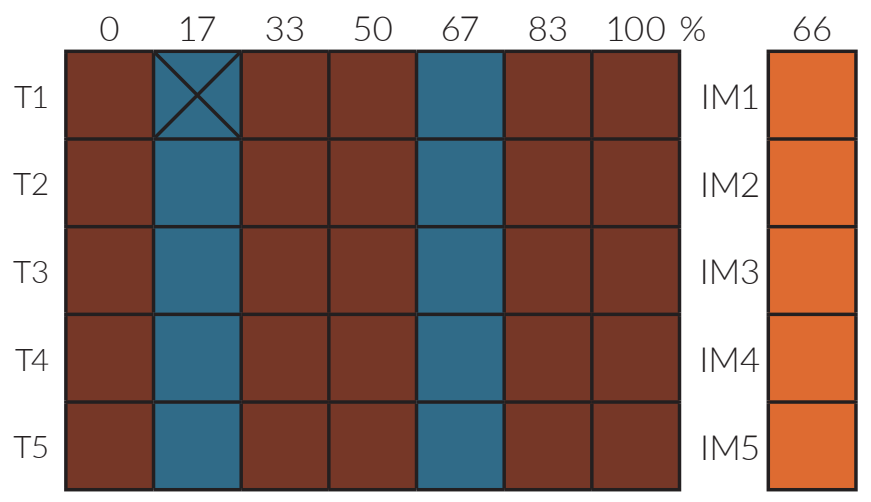

tion $A=\log _{10}(1 / R)$. All spectra were pre-processed using extended multiplicative scatter correction (EMSC), which is a pre-processing technique that corrects for undesired physical variations between the samples, such as surface geometry causing light scattering effects, ${ }^{17}$ while attempting to retain spectral variations caused by chemical differences between the samples. This is achieved by fitting each raw absorbance spectrum using ordinary least squares (OLS) fitting to a design matrix containing a constant term, a linear term consisting of a vector with all wavelengths included in the spectrum, a quadratic term containing a squared version of the wavelengths and a reference spectrum which is assumed to contain less scatter effects than each individual raw spectrum. The spectral correction is then carried out using

$$
S_{\text {Corr }}=\frac{S_{\text {Raw }}-b_{1}-b_{2} \cdot w-b_{3} \cdot w^{2}}{b_{4}}
$$

where $b_{1-4}$ are the regression coefficients, acquired with OLS, corresponding to the consecutive terms in the design matrix. $w$ is the vector of wavelengths involved, $S_{\text {Raw }}$ is the uncorrected spectrum and $S_{\text {Corr }}$ is the corrected spectrum.

The reference spectrum used during our EMSC was set to the average spectrum of all samples within the training data set, thereby avoiding information leakage between the training and validation data which were both processed using the same reference spectrum. For a more in-depth description of EMSC, the reader is referred to References 17 and 18.

\section{Wavelength selection and PLS regression}

Noisy and irrelevant parts of the spectra which were not correlated to the phosphorus content in any statistically meaningful way were removed by a backwards elimination algorithm. In backwards elimination, an initial regression model is created using all available variables, which in this case corresponds to wavelengths. The model is then created again $N$ times, where $N$ is the number of available wavelengths, with one of the available wavelengths inactivated each iteration until all wavelengths have been excluded once. The wavelength selection with the lowest root mean square error of cross-validation (RMSE $\mathrm{CV}_{\text {) }}$ of all the evaluated models then becomes the new startcase and the process starts over again recursively until no single wavelength inactivation can be made that improves the RMSE ${ }_{\mathrm{CV}}$. The backwards elimination algorithm utilised in this paper is summarised in pseudocode in Figure 3. All fitting of regression coefficients was done using a $C$ implementation of the Bidiag 2 partial least squares algorithm proposed in Reference 19, which was chosen due to its proven numerical stability and computational efficiency. The partial least squares (PLS) regression was configured to consider at most 25 components. Since backwards elimination can require several thousand regression models to be fitted before terminating, the spatial resolution of the hyperspectral data was down-sampled with regional pixel averaging to allow for

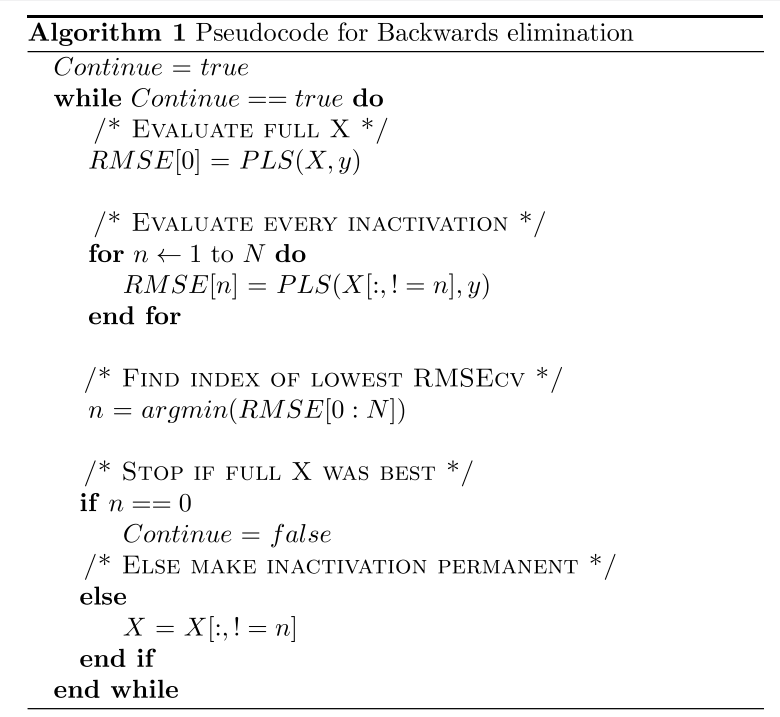

Figure 3. Pseudocode for the backwards elimination algorithm used to select relevant wavelengths. Crossvalidation loop omitted for clarity. The variable $\mathrm{N}$ symbolises the dynamically changing width of the design matrix $\mathrm{X}$. 
faster model development into 625 spectra $(25 \times 25)$ per hypercube.

\section{Results and discussion}

\section{Pre-processing}

Figure 4 shows the mean spectrum from all samples in the data set before (left) and after (right) being processed using EMSC. As can be seen to the right in Figure 4, the most significant change induced in the spectrum when varying the concentration of flame retardant compound appears to be in the $1900-2531 \mathrm{~nm}$ region. It is also noteworthy that the pressure-impregnated samples, which are displayed as dashed black lines in Figure 4, exhibit slightly different absorbance properties from the immersed samples at wavelengths shorter than $1500 \mathrm{~nm}$, which suggests that treatment method influences the absorbance properties of the sample in certain spectral regions. The absorbance difference between immersed and pressure-impregnated samples does, however, appear to become much less significant in the far-end of the spectrum.

\section{Wavelength selection}

The RMSE ${ }_{\text {cv }}$ kept decreasing as the backwards elimination algorithm ran until only 17 wavelengths remained active. As can be seen in Figure 5, illustrating the location of these wavelengths, all 17 active wavelengths were found in the 2400-2531 nm range. In terms of applying the model to the pressure-impregnated samples, the wavelengths selected by the backwards elimination algorithm seem to be among the most favourable, since the absorbance of all wood samples is similar in this wavelength region, regardless of treatment method. The number of
PLS components deemed optimal in the final variable selection with respect to $\mathrm{RMSE}_{\mathrm{CV}}$ was one, resulting in a model of low complexity. This identified wavelength range for determining phosphorus agrees with wavelength regions which have previously been reported in studies of phosphorus content in dried woody plant species $^{20}$ and in soil. ${ }^{21}$ The latter study in soil phosphorus concentration also found large regression coefficient values at other wavelengths, including the visible region. Gillon et al. ${ }^{22}$ studied phosphorus content in plant material with spectra in the visible (Vis) and NIR regions and found that most of the spectral information was found in the NIR part of the spectrum. Although optical properties in the $V$ is region of the spectrum were not measured for samples in this study, the supposed correlation between absorbance in the NIR region and phosphorous content agrees with the present findings.

\section{Model performance}

Figure 6 shows the phosphorus content from the ICP analysis versus the final regression model's prediction of the phosphorus content based on the samples absorbance for both the samples within the training data set (left) and the validation data set (right). The regression model's RMSE of the phosphorus content of the training data was $6055 \mathrm{mg} \mathrm{kg}^{-1}$ with a coefficient of determination of 0.90 . For the validation samples immersed in $17 \%$ and $67 \%$ chemical solution, the RMSE was $4297 \mathrm{mg} \mathrm{kg}^{-1}$ with a coefficient of determination of 0.87 . Similar good regression results have been obtained when predicting phosphorus in soil and ground plant material, ${ }^{21,22}$ whereas no previous study has been found using this technology on phosphorus in wooden surfaces.
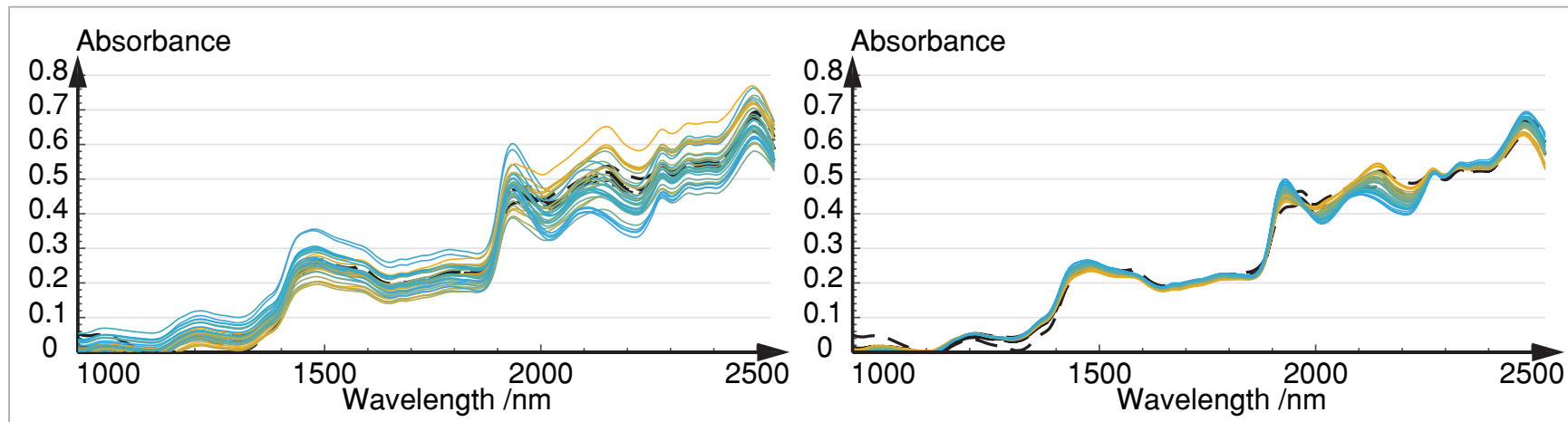

Figure 4. Mean spectrum of each sample in the dataset before (left) and after (right) being pre-processed using EMSC. Line colour indicates phosphorus content in the sample with yellow representing lower concentration and blue higher. Black dotted line indicates the spectrum from the pressure-impregnated samples. 


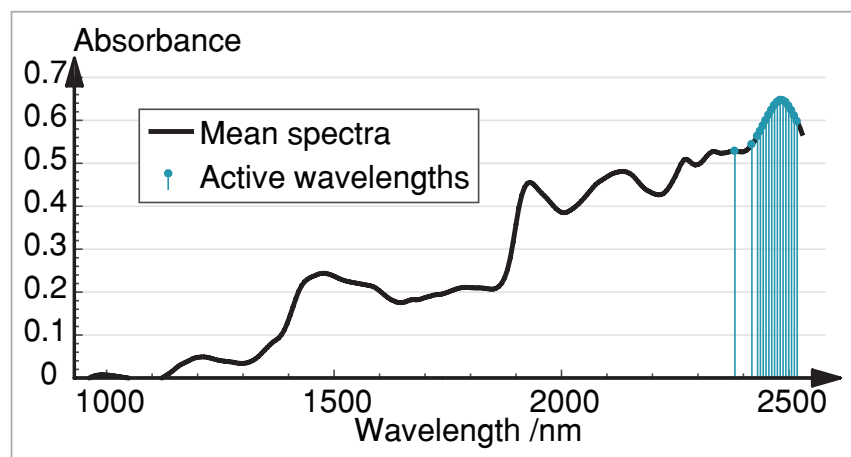

Figure 5. Wavelengths selected by backwards elimination. Blue lines represent wavelengths which were active after backwards elimination. The black curve represents the mean absorbance spectrum of the training dataset.

When using the model to predict the phosphorus content in the pressure-impregnated samples, the RMSE degraded to $24,394 \mathrm{mg} \mathrm{kg}^{-1}$ with a coefficient of determination of 0.87 . However, as can be seen both in the right part of Figure 6 and in Table 2 which provides a summary of the ICP results for all samples, this apparent collapse in RMSE can almost entirely be accredited to just one of the five impregnated samples, a sample which according to the ICP results supposedly has several times the phosphorus content compared to the other impregnated samples. It is unclear why sample IM1 has such an abnormally high phosphorous content considering that all impregnated samples were treated with the same solution. We can only speculate that it could be due to an exceptional deviation in sample density, an aggregation effect caused by an irregular surface curvature or a result of too high moisture conditions during storage. If the sample causing the RMSE collapse is disregarded as an outlier the RMSE of the four remaining impregnated samples becomes $8650 \mathrm{mg} \mathrm{kg}^{-1}$, which is more comparable to the immersed samples.

When reviewing the results in Figure 6 it is important to realise that, as is often the case in multivariate calibration with hyperspectral data, only the mean response value of each sample is known. Because multiple spectra share the same target value, it is therefore inevitable for the calibrated model to produce a distribution of errors around the target. In the absence of an alternative measurement technique which can be used to validate the chemical variations within each sample, the convention is to arrange the distribution of predicted values into two-dimensional images and visually determine if the in-sample variations display a credible pattern or not. Figure 7 shows the regression model's phosphorous prediction of every measured spectra for the immersed samples as a function of pixel position, arranged into chemical maps, with applied concentration of flame retardant increasing in the horizontal direction and the different wooden logs in the vertical direction. The model generally estimates a higher concentration of phosphorus in the earlywood regions than in the
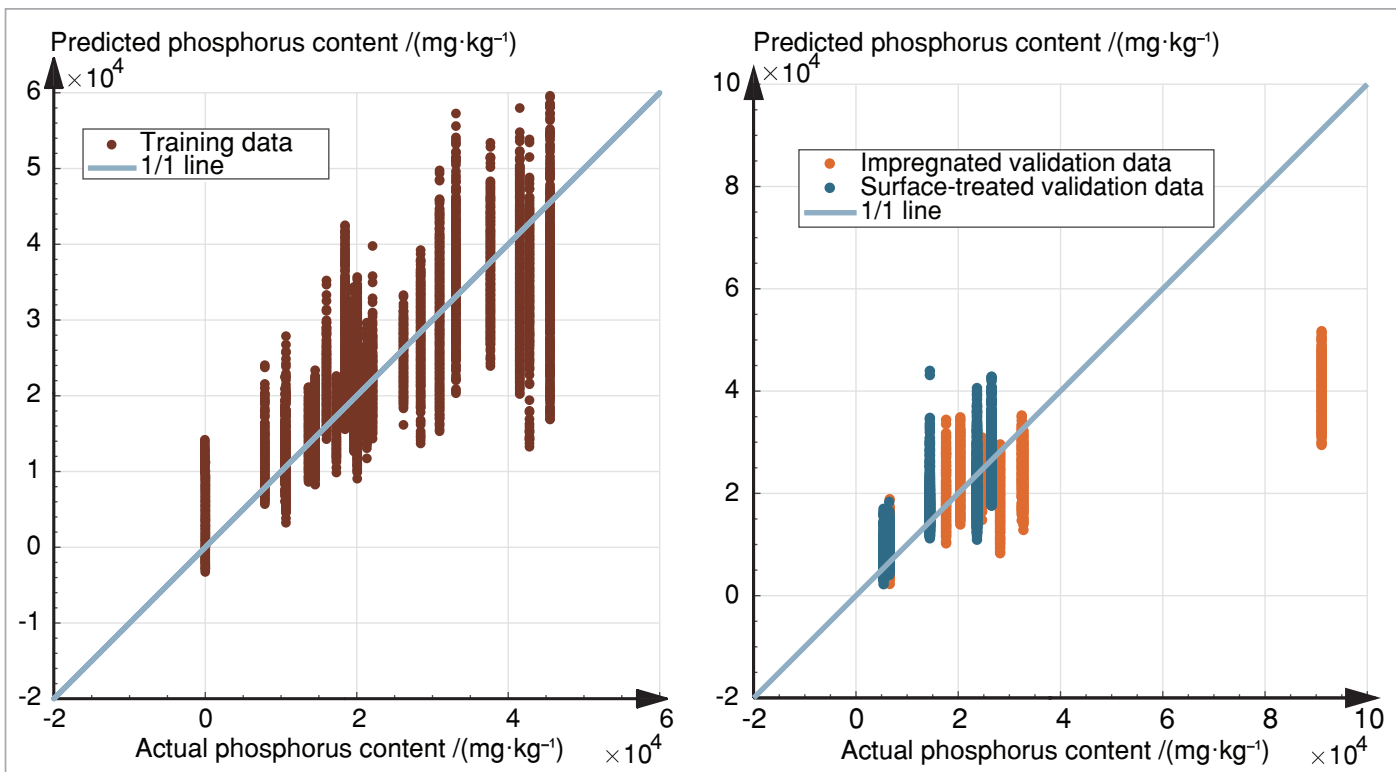

Figure 6. Regression plot of actual vs predicted phosphorus content for the training data (left) and the validation data (right). Each dot corresponds to one of the $25 \times 25$ spectra from the downsampled hypercubes. 
Table 2. Phosphorous concentration established by ICP for all samples together with mean, $\mu$, and standard deviation, $\sigma$, across replicates. All values are in the unit $\mathrm{mg} \mathrm{kg}^{-1}$.

\begin{tabular}{|c|c|c|c|c|c|c|c|}
\hline $\begin{array}{r}\text { Conc. } \\
\text { Sample }\end{array}$ & $\mathbf{0} \%$ & $\mathbf{1 7 \%}$ & $\mathbf{3 3 \%}$ & $\mathbf{5 0 \%}$ & $\mathbf{6 7 \%}$ & $\mathbf{8 3 \%}$ & $\mathbf{1 0 0 \%}$ \\
\hline T1 & 87 & 3207 & 7941 & 10,592 & 14,575 & 16,081 & 31,014 \\
\hline T2 & 72 & 5524 & 10,730 & 20,141 & 23,793 & 18,533 & 41,603 \\
\hline T3 & 90 & 6632 & 14,586 & 19,715 & 26,643 & 33,177 & 45,600 \\
\hline T4 & 20 & 6436 & 13,714 & 21,414 & 20,532 & 28,516 & 42,888 \\
\hline T5 & 80 & 6724 & 17,392 & 26,249 & 17,749 & 37,730 & 22,169 \\
\hline
\end{tabular}

\begin{tabular}{|c|c|}
\hline Conc. & \\
\hline Sample & $\mathbf{6 6 \%}$ \\
\hline IM1 & 91,188 \\
\hline IM3 & 24,769 \\
\hline IM4 & 32,871 \\
\hline IM5 & 32,542 \\
\hline
\end{tabular}

\begin{tabular}{|c|c|c|c|c|c|c|c|}
\hline$\mu$ & 70 & 5705 & 12,872 & 19,622 & 20,658 & 26,807 & 36,655 \\
\hline$\sigma$ & 29 & 1475 & 3639 & 5678 & 4773 & 9305 & 9811 \\
\hline
\end{tabular}

\begin{tabular}{|l|l|}
\hline$\mu$ & 41,937 \\
\hline$\sigma$ & 27,733 \\
\hline
\end{tabular}

latewood regions. This makes intuitive sense, since the earlywood regions of Norway Spruce have lower density and are more susceptible to absorbing liquids, but this is a phenomenon which, to the authors' knowledge, has never been demonstrated before with flame retardant treatments. Thumm et al. have previously demonstrated that resin affects the spectral signature of wood around the $1180 \mathrm{~nm}$ and $1370 \mathrm{~nm}$ regions. ${ }^{15}$ As can be seen on sample T1 17\% in Figure 7, which depicts the modelled phosphorous of the resin covered sample, it is clear that

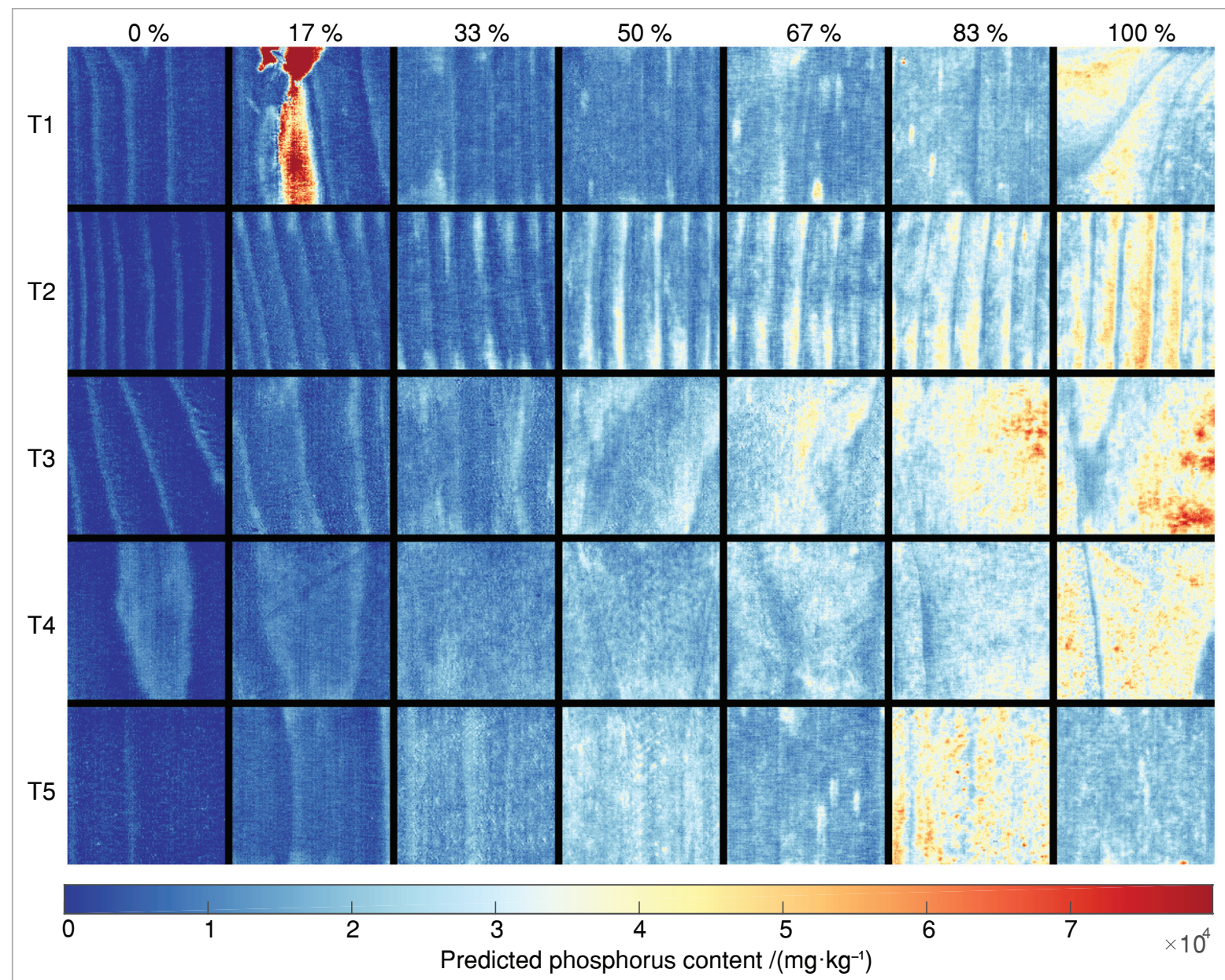

Figure 7. Prediction of phosphorus content for every immersed sample in the data set. Sample T1 $17 \%$ illustrates the outlier sample covered in resin which was not considered when evaluating the performace of the model. 
resin also alters the spectra in the $2400-2531 \mathrm{~nm}$ range, which in this case causes a local misclassification of phosphorous. If the spectral signature of resin was studied further it is possible that a different wavelength subset could be identified which would allow a model to accurately predict phosphorous whilst being unaffected by the presence of resin.

Figure 8 shows the chemical maps for the pressureimpregnated samples. As can clearly be seen in Figure 8, the model does estimate, in accordance with the ICP analysis, that one of the pressure-impregnated samples has a substantially higher surface concentration of phosphorus than the others. The precise quantity of phosphorus reported by the ICP analysis and the regression model does however differ substantially as shown in Figure 6. If indeed the phosphorus concentration reported by the ICP analysis for this sample is valid, it is not surprising that the regression model struggles in its estimation since it has a response value far higher than anything used in the calibration of the model.

\section{Conclusions}

We demonstrated that NIR hyperspectral imaging together with PLS regression can be used as a novel non-destructive tool for surveying the current condition of phosphorus-based flame retardant chemical compounds, both surface-applied and pressure-impregnated into, samples of Norway spruce. In most cases our model was able to predict the phosphorus content in wood surfaces with a high degree of accuracy with an $R^{2}$ of 0.87 on independent validation samples. However, since the method works by measuring how light is reflected off the surface, it is vulnerable to surface defects, such as resin stains, which locally conceals the true physical properties of the wood

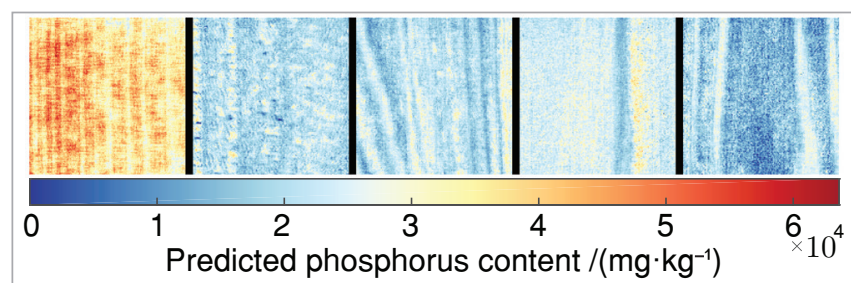

Figure 8. Prediction of phosphorus content of the pressure-impregnated samples. Left-most square represents the sample which, according to the ICP analysis, had a substantially higher phosphorus content compared to the other pressure-impregnated samples. surface and can cause misleading phosphorus estimates. When using the model to estimate the spatial distribution of phosphorus in our samples, the chemical uptake does not occur entirely evenly throughout the wood. Instead, the highest concentration of phosphorus was generally found in the earlywood regions of the surfaces.

We identified that 2400-2531 nm appears to be a key wavelength region when it comes to estimating phosphorus. Since this region was at the limit of what our instrument could measure, further studies should investigate the possibility of estimating phosphorus using an instrument capable of detecting longer wavelengths into the infrared region.

\section{Acknowledgement}

The authors wish to thank Akzo Nobel Coating AS for supplying the flame retardant chemical along with the pressure-impregnated samples used in the study. This work has been funded by the Norwegian Research Council in the project "WOOD/BE/BETTER" code 225345.

\section{References}

1. J. Hildebrandt, N. Hagemann and D. Thrän, "The contribution of wood-based construction materials for leveraging a low carbon building sector in Europe", Sustain. Cities Soc. 34, 405-418 (2017). doi: https://doi.org/10.1016/j.scs.2017.06.013

2. M. Herczeg, D. McKinnon, L. Milios, I. Bakas, E. Klaassens, K. Svatikova and O. Widerberg, Resource Efficiency in the Building Sector. ECORYS Nederland BV, Rotterdam (2014).

3. "Woodworking - Growth - European Commission", Growth (2017). https://ec.europa.eu/growth/sectors/ raw-materials/industries/forest-based/woodworking_en [Accessed: 14 September 2017].

4. Technical Guideline for Fire Safety in Timber Buildings. RISE Research Institutes of Sweden (2017). https:// www.sp.se/FSITB [Accessed: 14 September 2017].

5. W.D. Ellis and R.M. Rowell, "Flame-retardant treatment of wood with a diisocyanate and an oligomer phosphonate", Wood Fiber Sci. 21(4), 367-375 (1988). https://wfs.swst.org/index.php/wfs/article/ view/546 
6. P. Gou, E. Santos-Garcés, M. Høy, J. Wold, K. Liland and E. Fulladosa, "Feasibility of NIR interactance hyperspectral imaging for on-line measurement of crude composition in vacuum packed dry-cured ham slices", Meat Sci. 95(2), 250-255 (2013). doi: https:// doi.org/10.1016/j.meatsci.2013.05.013

7. A. Sandak, J. Sandak and R. Meder, "Tutorial: assessing trees, wood and derived products with near infrared spectroscopy: hints and tips", J. Near Infrared Spectrosc. 24(6), 485-505 (2016). doi: https://doi.org/10.1255/jnirs.1255

8. A. Sandak, J. Sandak and M. Riggio, "Assessment of wood structural members degradation by means of infrared spectroscopy: an overview", Struct. Contr. Health Monitor. 23(3), 396-408 (2016). doi: https:// doi.org/10.1002/stc.1777

9. M. Schwanninger, J. Rodrigues and K. Fackler, "A review of band assignments in near infrared spectra of wood and wood components", J. Near Infrared Spectrosc. 19(5), 287-308 (2011). doi: https://doi. org/10.1255/jnirs.955

10. H. Kobori, N. Gorretta, G. Rabatel, V. Bellon-Maurel, G. Chaix, J. Roger and S. Tsuchikawa, "Applicability of vis-NIR hyperspectral imaging for monitoring wood moisture content (MC)", Holzforschung 67(3), 307-314 (2013). doi: https://doi.org/10.1515/ hf-2012-0054

11. A. Thumm, M. Riddell, B. Nanayakkara, J. Harrington and R. Meder, "Near infrared hyperspectral imaging applied to mapping chemical composition in wood samples", J. Near Infrared Spectrosc. 18(6), 507-515 (2010). doi: https://doi.org/10.1255/jnirs.909

12. T. Lestander, M. Finell, R. Samuelsson, M. Arshadi and M. Thyrel, "Industrial scale biofuel pellet production from blends of unbarked softwood and hardwood stems-the effects of raw material composition and moisture content on pellet quality", Fuel Process. Technol. 95, 73-77 (2012). doi: https://doi. org/10.1016/j.fuproc.2011.11.024

13. I. Burud, K. Smeland, K. Liland, J. Sandak, A. Sandak, L. Gobakken and T. Thiis, "Near infrared hyperspectral imaging in transmission mode: assessing the weathering of thin wood samples", J. Near Infrared Spectrosc. 24(6), 595-604 (2016). doi: https://doi. org/10.1255/jnirs.1253

14. R. Meder, J. Brawner, G. Downes and N. Ebdon, "Towards the in-forest assessment of Kraft pulp yield: comparing the performance of laboratory and handheld instruments and their value in screening breeding trials", J. Near Infrared Spectrosc. 19(5), 421-429 (2011). doi: https://doi.org/10.1255/jnirs.954

15. A. Thumm and M. Riddell, "Resin defect detection in appearance lumber using 2D NIR spectroscopy", Eur. J. Wood Wood Prod. 75(6), 995-1002 (2017). doi: https://doi.org/10.1007/s00107-017-1188-5

16. G. Ogner, T. Wickstrøm, G. Remedios, S. Gjelsvik, G.R. Hensel, J.E. Jacobsen, M. Olsen, E. Skretting and B. Sørlie, The Chemical Analysis Program of the Norwegian Forest Research Institute 2000. Norwegian Forest Research Institute, Ås, Norway, p. 23 (1999).

17. H. Martens and E. Stark, "Extended multiplicative signal correction and spectral interference subtraction: new preprocessing methods for near infrared spectroscopy", J. Pharmaceut. Biomed. Anal. 9(8), 625-635 (1991). doi: https://doi. org/10.1016/0731-7085(91)80188-F

18. N. Afseth and A. Kohler, "Extended multiplicative signal correction in vibrational spectroscopy, a tutorial", Chemometr. Intell. Lab. Sys. 117, 92-99 (2012). doi: https://doi.org/10.1016/j.chemolab.2012.03.004

19. А. Björck and U. Indahl, "Fast and stable partial least squares modelling: a benchmark study with theoretical comments", J. Chemometr. 31(8), e2898 (2017). doi: https://doi.org/10.1002/cem.2898

20. C. Petisco, B. García-Criado, B. Vázquez de Aldana, I. Zabalgogeazcoa, S. Mediavilla and A. GarcíaCiudad, "Use of near-infrared reflectance spectroscopy in predicting nitrogen, phosphorus and calcium contents in heterogeneous woody plant species", Anal. Bioanal. Chem. 382(2), 458-465 (2005). doi: https://doi.org/10.1007/s00216-004-3046-7

21. N. Dhawale, V. Adamchuk, R.A.V. Rossel, S. Prahser, A.A. Ismail and J.K. Whalen, "Predicting extractable soil phosphorus using visible/near-infrared hyperspectral soil reflectance measurements", The Canadian Society for Bioengineering 2013 Annual Meeting, Saskatoon, Saskatchewan, 7-10 July 2013, Paper No. CSBE13-047 (2013).

22. D. Gillon, C. Houssard and R. Joffre, "Using nearinfrared reflectance spectroscopy to predict carbon, nitrogen and phosphorus content in heterogeneous plant material", Oecologia 118(2), 173-182 (1999). doi: https://doi.org/10.1007/s004420050716 